\title{
GAIA Level 2 Antepartum Stillbirth
}

National Cancer Institute

\section{Source}

National Cancer Institute. GAIA Level 2 Antepartum Stillbirth. NCI Thesaurus. Code C128019.

GAIA Level 2 Antepartum Stillbirth is defined by four criteria: first, delivery of an infant with no of signs of life at birth, including the following: no spontaneous movements, no umbilical cord pulse, no heartbeat, no respirations, and an Apgar score of 0 at both 1 and 5 minutes. Determination of the absence of signs of life is made by physical examination after delivery, with or without electronic monitoring of heart rate, respiratory rate, and pulse oximetry; second, one or more of the following three requirements must be met: a) Maternal report of the absence of fetal movement for 24 hours or more; b) Maternal physical examination confirming the absence of fetal movement; c) Auscultation for fetal heart tones using electronic devices (e.g. handheld Doppler or fetal heart rate monitor) or non-electronic devices (e.g. stethoscope, fetoscope or Pinard horn) that documents the absence of a fetal heartbeat; third, one or more of the following two requirements must be met: a) Attended delivery followed by a post-delivery physical examination of the fetus that is performed by specialist or qualified trained practitioner appropriate to the health care setting, and that is consistent with antepartum death; b) fetal/placental pathology report that is consistent with antepartum death; fourth, gestational age within the pre-defined range for selected stillbirth definition, as assessed by maternal and/or fetal parameters (level 1-2 in GA assessment algorithm). 